\title{
SNR performance comparison of Dual-Layer Detector and Dual-kVp spectral CT
}

\author{
B. J. Heismann and S. Wirth
}

\begin{abstract}
Tube-based dual-kVp and detector-based duallayer sandwich detector CT systems can be used to generate spectral CT data. We investigate the influence of their energy weighting functions on the performance of reconstructed base material coefficient images and projections. A Monte-Carlo (MC) simulation of the noise propagation for typical water and bone CT projections is used. We find a large difference in the SNR of the reconstructed coefficient projections. Dual-kVp scanning methods yield a factor of 2 better SNR in the coefficient projections than dual-layer detector based CT. This can be explained by the degree of overlap of the weighting functions, especially for low-energy $\mathrm{X}$-ray quanta.
\end{abstract}

\section{INTRODUCTION}

$\mathrm{D}$ ual-kVp and dual-layer detector CT scans are two approaches to dual-energy spectral CT. We can achieve dual-kVp scans by tube voltage switching for alternating projections [1], two scans at different tube voltage settings or

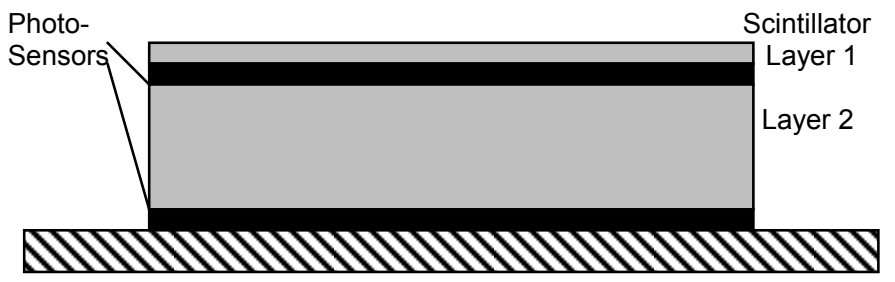

Fig. 1: Schematic dual-layer detector set-up.

dual-source CT [2]. On the other hand dual-layer sandwich detectors $[3,4]$ can be embedded into a conventional CT system. Basically two scintillator detectors are placed on top of each other (Fig. 1) to detect different portions of the incoming spectrum.

In this paper we compare the base-material decomposition performance of the two approaches. For typical CT projections of bone and water attenuating cross-sections (Fig. 2 ), the SNR of the reconstructed coefficients is calculated by a Monte Carlo (MC) method established in [5].

B. J. Heismann is with the Compuited Tomography Division of Siemens Medical Solutions, Siemensstr. 1, 91301 Forchheim, Germany (e-mail: bjoern.heismann $<$ at $>$ siemens.com).

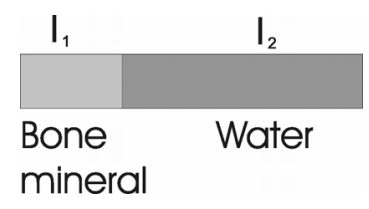

Fig. 2: Model geometry of bone mineral and water attenuator.

\section{THEORY}

The projection $P$ in a CT measurement is given by:

$$
P=\frac{I}{I_{0}}=\int_{E} w(E) e^{-\int \kappa(E, \vec{r}) d \vec{r}} d E
$$

with: $\quad I:=$ measured intensity with absorber,

$I_{0}:=$ measured intensity without absorber,

$\kappa(E, \boldsymbol{r}):=$ spectral attenuation coefficient.

The weighting function $w(E)$ is given by

$$
w(E)=\frac{S(E) D(E)}{\int_{E} S(E) D(E) d E}
$$

with: $S(E):=$ X-ray tube emission spectrum,

$D(E):=$ detector sensitivity.

For a dual-energy scan we generate two sets of projections $P_{1}, P_{2}(1)$ with different spectral weightings, e.g. $w_{l}(E), w_{2}(E)$. The $w_{i}$ are calculated by taking $S(E)$ from tabulated spectra, e.g. [6], and simulating the detector X-ray detection physics to obtain $D(E)$ [7]. The resulting $w_{i}$ for a typical dual-kVp scan $(80 \mathrm{kV} \& 140 \mathrm{kV})$ and dual-layer scan $(0.2 \mathrm{~mm} \mathrm{\&} 1.4 \mathrm{~mm}$ GdOS scintillator) are shown in Figs. 3a, $3 \mathrm{~b}$.

The base material decomposition [8] converts the dualenergy projections $P_{1}, P_{2}$ into two coefficient projections $B_{1}$, $B_{2}$ by solving

$$
\left(\begin{array}{l}
P_{1} \\
P_{2}
\end{array}\right)=\left(\begin{array}{l}
\int_{E} w_{1}(E)\left(e^{-F_{1}(E)}\right)^{B_{1}}\left(e^{-F_{2}(E)}\right)^{B_{2}} d E \\
\int_{E} w_{2}(E)\left(e^{-F_{1}(E)}\right)^{B_{1}}\left(e^{-F_{2}(E)}\right)^{B_{2}} d E
\end{array}\right)
$$

with: $F_{1}, F_{2}:=$ base material attenuation functions.

Since reconstructing the coefficient images $b_{l}(\boldsymbol{r}), b_{2}(\boldsymbol{r})$ from a sinogram set $\left\{B_{1}\right\},\left\{B_{2}\right\}$ is a linear process, the SNR of 


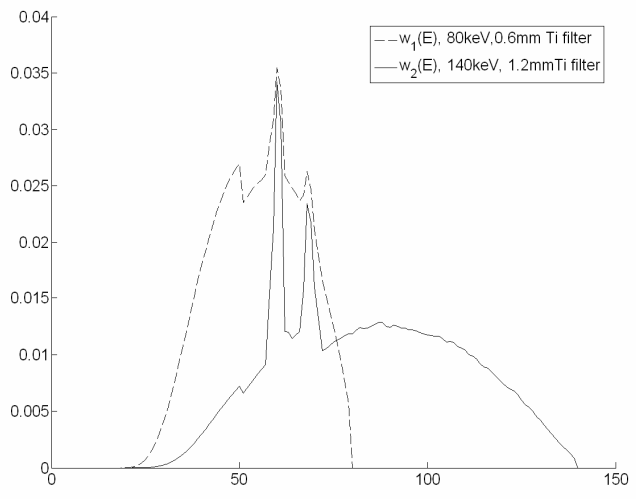

Fig. 3a

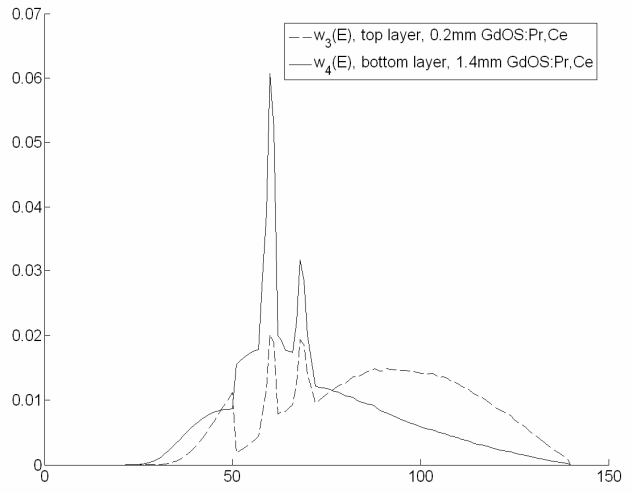

Fig. 3b

Fig.3a: Energy weighting functions $w_{l}(E), w_{2} / E$ ) for a typical dual-kVp scan with $80 \mathrm{kV}$ and $140 \mathrm{kV}$ tube settings.

Fig 3b: $w_{3}(E), w_{4}(E)$ for a typical dual-layer detector scan with $0.2 \mathrm{~mm}$ and $1.4 \mathrm{~mm}$ top and bottom layer GdOS:Pr scintillator detector.

$B_{1}, B_{2}$ is also a direct measure for the coefficient image noise. It is thus a good figure of merit for the performance comparison of the dual-energy measurement methods.

\section{Simulation}

We perform a Monte-Carlo (MC) simulation of the noise transfer with the following steps, see also [5]:

1. The dual-energy CT data of the projections $P_{1}, P_{2}$ is calculated according to (1) with the $w_{i}(E)$ of Fig. $3 \mathrm{a}, \mathrm{b}$ and using the attenuation geometry of Fig. 2.

2. For the $\mathrm{MC}$ simulation of the probability distributions of $B_{1}, B_{2}$ we repeat the following procedure $M$ times:

- First, Poisson noise is added to the ideal projections $P_{1}, P_{2}$ of step 1 with a $\sigma$-value of

$$
\sigma\left(P_{i}\right)=\sigma\left(\frac{N_{i}}{N_{i, 0}}\right)^{\sigma\left(N_{i, 0}\right) \text { negl. }}=\sqrt{\frac{P_{i}}{N_{i, 0}}}
$$

The quantum numbers $N_{i}$ and $N_{i, 0}$ are given by the intensities $I_{i}$ and $I_{i, 0}$ of the two measurements $i=1,2$ multiplied with the sensor area and integration time. Since the $I_{i, 0}$ measurements are averaged significantly in practical $\mathrm{CT}$, the noise contribution $\sigma\left(N_{i, 0}\right)$ is negligible in (3).

- Secondly, the $B_{1}, B_{2}$ are calculated for each $P_{1}, P_{2}$.

3. Finally we compare the SNR of $B_{1}, B_{2}$ to the SNR of the combined projection $P=\left(N_{1}+N_{2}\right) /\left(N_{l, 0}+N_{2,0}\right)$ :

$$
\operatorname{SNR}(P)=\sqrt{P_{1} N_{1,0}+P_{2} N_{2,0}}
$$

The results in this paper were obtained with $F_{1}, F_{2}=$ Water and bone mineral (CaHOP) base functions, $L=30 \mathrm{~cm}, l_{l}=$ $0 \ldots 4.5 \mathrm{~cm}$ of dense bone material, $N_{0}=0.5 \cdot 10^{6}, N_{1,0}=N_{2,0}=$ $N_{0} / 2, w_{1}, w_{2}$ and $w_{3}, w_{4}$ according to Fig. $3 \mathrm{a}, \mathrm{b}, M=1000$ $\mathrm{MC}$ cycles.

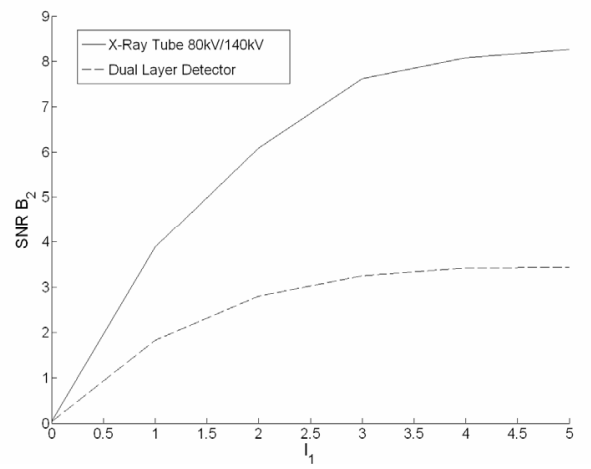

Fig.5 Simulated $\operatorname{SNR}\left(B_{2}\right)$ as a function of bone attenuator length $l_{l}$.

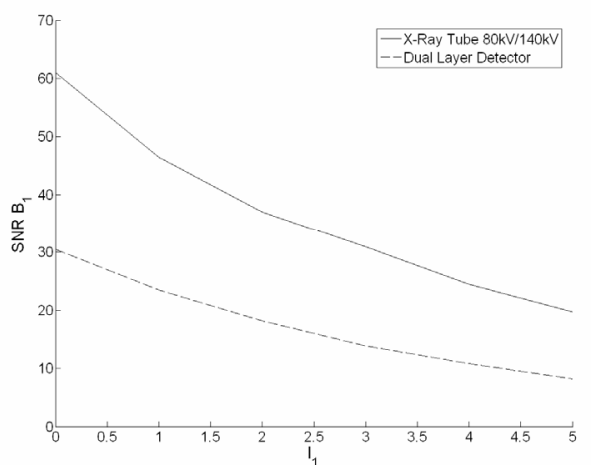

Fig. 4 Simulated SNR $\left(B_{l}\right)$ as a function of bone attenuator length $l_{l}$.

\section{RESUltS AND DiscUSSION}

Figs. 4 and 5 show the resulting $\operatorname{SNR}\left(B_{1}\right)$ and $\operatorname{SNR}\left(B_{2}\right)$ for dual-kVp and dual-layer spectral CT as a function of the bone 
length $l_{l}$ in the attenuator (Fig. 2). $\operatorname{SNR}\left(B_{I}\right)$ decreases and $\operatorname{SNR}\left(B_{2}\right)$ increases with $l_{l}$. The $\operatorname{SNR}\left(B_{l}\right)$ is about 1.8 to 2 times better for the dual-kVp CT. For $\operatorname{SNR}\left(B_{2}\right)$ we obtain a factor of 2 to 2.6. This is explained by the weighting function pairs $w_{1}, w_{2}$ and $w_{3}, w_{4}$ shown in Figs. 3a, 3b. For dual-kVp scanning the overlap of the $w_{1}, w_{2}$ is about $45 \%$, for dual-layer scanning about $64 \%$. The stronger overlap reduces differentiation in $P_{1}, P_{2}$ and noise in $P_{1}, P_{2}$ leads to larger noise in $B_{1}, B_{2}$ for dual-layer CT. Since low-energy photons contribute more contrast to $P_{l}, P_{2}$ due to Photo-Effect attenuation being $\propto \mathrm{E}^{-3}$ especially the dual-layer weighting overlap for low-energies increases this effect.

Note that the dual-layer detector might be optimized by using a low- $Z$ scintillator material with less high-energy absorption in the first layer [9]. However, this is limited by the requirement to have a balanced number of absorbed quanta in both layers for good SNR performance and the requirement to have matching afterglow characteristics in both layers. We conclude that the factor of 2 in SNR advantage of dual-kVp spectral CT will be hard to overcome with duallayer detectors.

\section{BIBLIOGRAPHY}

[1] W.A. Kalender, W.H. Perman, J.R. Vetter, E. Klotz, Med Phys 13, 334-339 (1986)

[2] T.G. Flohr, et al., Eur Radiol. 2006 Feb; 16(2):256-68.

[3] B. J. Heismann, S. Wirth, S. Janssen, Q. Spreiter, Proc. of SPIE, 5368, 52-59 (2004)

[4] R. Carmi, G. Naveh. A. Altman.. IEEE Medical Imaging Conference Record (2005)

[5] B.J. Heismann, M11-366, IEEE Medical Imaging Conference Record (2005)

[6] J.M. Boone, J.A. Seibert, Med. Phys. 24, 1661-1670 (1997)

[7] S. Wirth, K. Pham-Gia, W. Metzger, B.J. Heismann, M11-212, IEEE Medical Imaging Conference

Record (2006)

[8] R.E. Alvarez. A. Macovski, Phys. Med Biol. 21(5):733-44 (1976)

[9] J. E. Tkaczyk et al., 6510-08, SPIE Medical Imaging Conference (2007) 